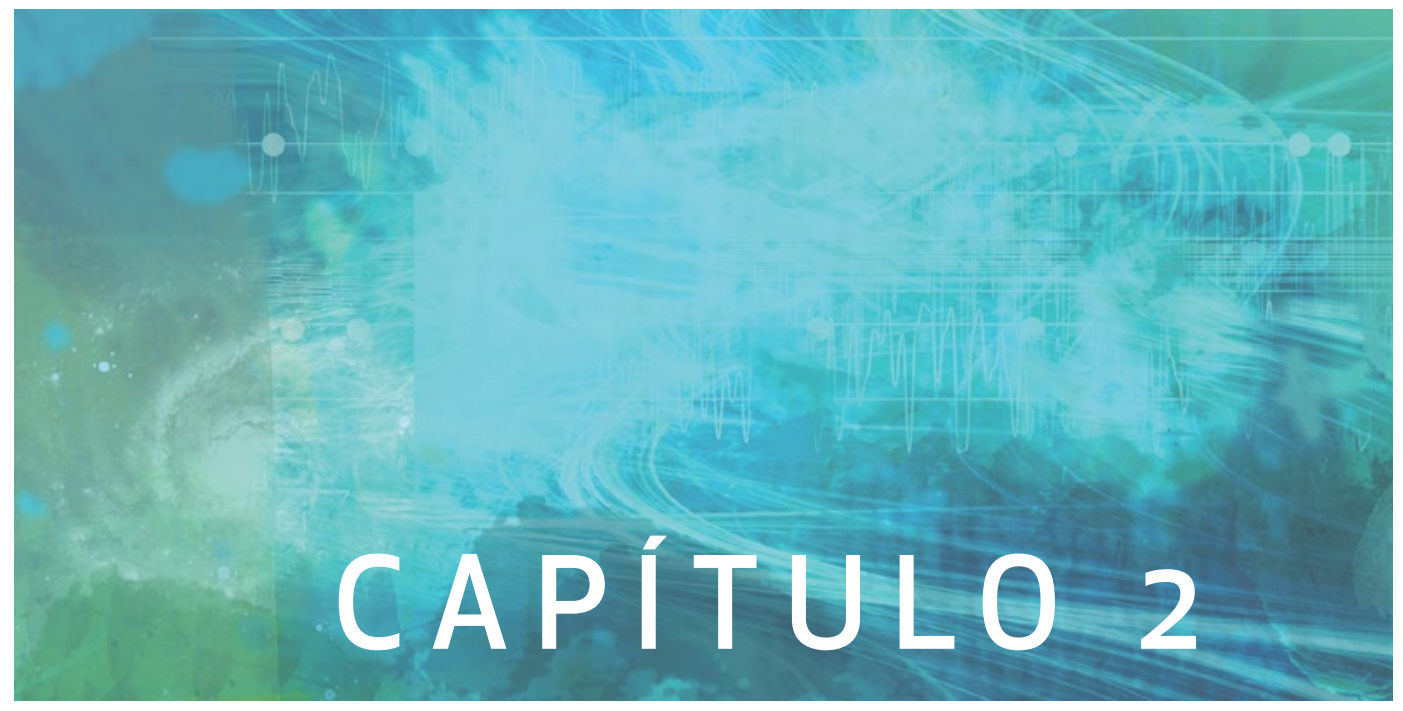





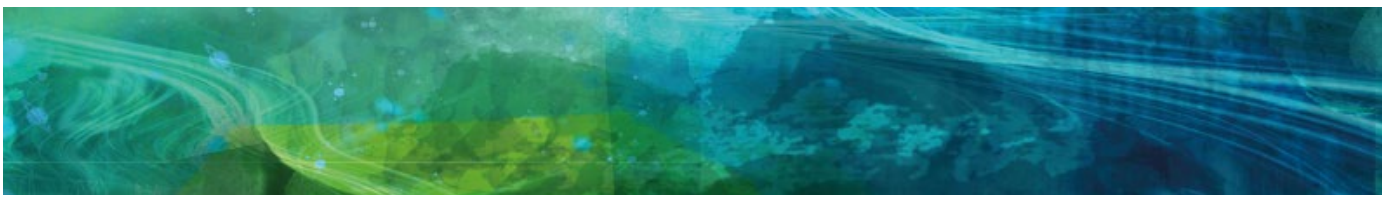

\section{El control interno del Estado colombiano: un instrumento administrativo que facilita la competitividad del sector productivo}

Germán Fernando Medina Ricaurte ${ }^{5}$

5 Magister en Administración de Organizaciones (UNAD, Colombia). Especialista en Gestión Pública (ESAP, Colombia). Especialista en Pedagogía del Aprendizaje Autónomo (UNAD, Colombia). Economista (UGC, Colombia). Docente de carrera de la Universidad Nacional Abierta y a Distancia (UNAD). Correo electrónico: german. medina@unad.edu.co 


\section{Resumen}

Este capítulo aborda temas como el control interno de las entidades del Estado y la competitividad de las empresas de derecho privado. Analizando la relación que existe entre ellas, con el fin de observar cómo el sector público puede aportar al sector productivo de la economía, para que esta se desarrolle adecuadamente.

La metodología utilizada ha sido la revisión del marco teórico que define el control, para posteriormente abordar el control interno en las entidades del Estado, partiendo de la Constitución Política, las leyes y decretos, para articularlo con herramientas de gestión que aporten a la competitividad de las empresas y la economía en general. El capítulo tiene como resultados la articulación que debe existir entre las empresas del gobierno y las empresas privadas, alrededor de la competitividad, con el fin de facilitar los procesos para que estos sean ágiles y acordes con las nuevas necesidades de los mercados locales y globales.

Como valor agregado se presenta una propuesta para que se integren herramientas de gestión administrativa que se han desarrollado en el sector privado y que se podrían adaptar al sector público, para que mejore sus estructuras administrativas, y de esta forma sean más competitivas y aporten al proceso productivo del país.

Palabras claves: control, productividad, empresa, inteligencia competitiva, enfoque sistémico 


\section{Introducción}

El presente capítulo se elaboró con base en la revisión y análisis de los documentos normativos que soportan los procesos mediante los cuales se origina la implementación del sistema de control interno en las entidades del Estado colombiano, como una herramienta que facilita el control y la gestión de lo público dentro de los principios de igualdad, moralidad, eficiencia, economía, celeridad, imparcialidad, publicidad y valoración de costos ambientales.

Se aborda el concepto y gestión de las empresas públicas como una organización o empresas que participan en un mercado, en donde se articulan las empresas privadas, generando sinergias que se dinamizan dentro del contexto global, lo que exige que las empresas del Estado deban ser competitivas para que correspondan a la nueva realidad del mercado global. Dentro de este concepto se procederá a observar la reglamentación sobre sistemas de control aplicada a las entidades del Estado, haciendo énfasis en que el control interno de las entidades del Estado es un componente que debe aportar elementos que faciliten la administración y la gestión del sector productivo de la economía.

Los ciudadanos que interactúan con el Estado lo suelen hacer de diferentes formas: algunas veces como agentes particulares, que se relacionan en diferentes escenarios sociales, personales y económicos; aunque también existen otras relaciones, por ejemplo, cuando prestan sus servicios como servidores públicos o contratistas del Estado. Cualquiera que sea el rol que asuma el ciudadano para lograr una convivencia adecuada dentro de la sociedad, deberá conocer, acatar y respetar el orden constitucional establecido en el país en el que reside. En Colombia, la actual Constitución Política fue promulgada en el año 1991. En este documento están establecidos los derechos y deberes de los ciudadanos, así como la estructura del Estado colombiano y el régimen económico vigente.

Las nuevas teorías de desarrollo endógeno sustentan la idea de que los países o regiones encierran, en su propia dinámica, la clave para un crecimiento sostenido. Esto significa que, mediante la aplicación de políticas adecuadas, se 
pueden generar las condiciones propicias para el desarrollo de las economías, siempre propendiendo por un bienestar de los ciudadanos, que le confían al Estado la administración de los bienes públicos.

Las empresas modernas tienen un gran contacto con el Estado. Y esto se evidencia a través de la relación de pago de tributos como el impuesto a la renta, el impuesto de avisos, tableros y complementarios, impuestos de importaciones y exportaciones, licencias y permisos, entre muchos otros trámites que las empresas deben realizar ante diferentes organismos del estado. Por este motivo, y para que exista una relación armoniosa entre las empresas y el Estado, se deben desarrollar los procesos administrativos con la mayor eficiencia y evitar que los errores generen traumatismos en la prestación de los servicios, para lo cual es necesario que el Estado cuente con mecanismos de control que faciliten la gestión de las entidades.

En el ejercicio de su actividad diaria o en el diseño de su estrategia, el empresario no puede olvidar la existencia del Estado, especialmente en la sociedad contemporánea. Las formas de actuación del Estado cambian con el tiempo, pero todo parece indicar que el Estado mismo no desaparece. Al contrario, en las últimas décadas su peso no ha hecho más que crecer; y es lógico que se cuestione si ese crecimiento tiene límites, y, si los tiene, dónde están. Para ello hay que preguntarse cuál es la razón de ser de las diversas actividades del Estado.

Se observa que las relaciones entre el sector público y el empresario privado abarcan una amplia gama de sentimientos que van de la cordialidad al enfrentamiento, pasando por la complicidad y la indiferencia. Que esas relaciones sean de uno u otro carácter depende de varios factores: de la actividad de que se trate (que puede merecer más o menos atención por parte de los poderes públicos), de la afinidad con el gobierno (relación entre los funcionarios del gobierno con los representantes de las empresas). A menudo se habla de la conveniencia que se genera cuando el Estado y la empresa trabajan juntos, pero esto no siempre es posible. Esta situación se replica en la mayoría de los países en lo que concierne a la relación Estado-empresa, lo que hace que surja un tema crítico que tiene que ver con la fiscalización y control de las entidades públicas. 
La relación de la empresa con el Estado se hace más evidente cuando se observa la regulación que existe para la contratación y el pago de la mano de obra que participa en el proceso productico; además, de la regulación sobre patentes y su cumplimiento dentro del contexto nacional. Estos son unos cuantos ejemplos de la estrecha relación entre el Estado y las empresas privadas.

De acuerdo con lo planteado, es muy importante que los empresarios, como miembros de la sociedad, en el momento de la toma de decisiones tengan en cuenta los efectos que estas pueden tener en el actual orden constitucional, con el fin de que puedan aprovechar adecuadamente las ventajas que ha establecido el Estado colombiano para el desarrollo de la libre empresa y la protección a la propiedad privada. Así se puede evitar que, por desconocimiento de las relaciones con el Estado, se tomen decisiones con efectos negativos para la sociedad, pues estas podrían además generar una sanción por parte del Estado.

Cuando los empresarios tienen pleno conocimiento de las actividades del Estado y de sus procesos, es posible que estos puedan participar, con gran probabilidad de tener éxito, en los diferentes procesos licitatorios que adelanta el Gobierno para la adquisición de bienes y servicios; y de esta forma contribuir a que el Estado pueda cumplir con la función que ha sido establecida en la Constitución Política de Colombia. Es por este motivo que el Estado debe desarrollar procesos eficientes, con el objetivo de beneficiar el desempeño de las empresas privadas.

\section{Revisión de literatura}

\section{Globalización}

La sociedad contemporánea identifica la globalización como una actividad económica, tecnológica y cultural que se manifiesta a nivel global, y se refleja en unos procesos de comunicación que generan una interdependencia entre los países en los procesos mercantiles. Esto trae consigo cambios en las relaciones socioeconómicas y políticas en el globo. 
La dinámica global es el escenario en el que las empresas participan en mercados globales con una visión local de los procesos económicos, para lo cual necesitan contar con instituciones estatales que permitan unas dinámicas de negocios ágiles, es decir, con la menor cantidad de barreras no naturales. Por otro lado, el Estado moderno, como regulador de normas y procesos, tiene una importante participación en las actividades comerciales, tanto en el comercio interior como en el comercio exterior.

Las regulaciones comerciales en Colombia están establecidas en el código de comercio, la verificación del cumplimiento de esta norma está a cargo de organismos privados y públicos. Dentro de los organismos privados que realizan controles a las empresas se encuentran la Cámara de Comercio y el Icontec, entre otros. A nivel público, el control de las regulaciones a nivel municipal, tanto tributarias como de permisos, están a cargo de instituciones como la Secretarias de Hacienda, Salud, Hábitat y Medio Ambiente, entre otros organismos; a nivel nacional, la Dian, los ministerios del gobierno nacional y el Sena, entre otros.

Para generar condiciones adecuadas que permitan el desarrollo de la empresa nacional, facilitando la gestión de sus procesos a nivel nacional e internacional, se requiere de un Estado que sea a su vez fuerte y ágil, que organice en forma eficiente las relaciones con el sector productivo, para que no se presenten demoras innecesarias en la relación público - privado que puedan generar costos adicionales a las empresas, por falta de operatividad de las entidades públicas.

Dentro de los elementos que hacen parte de la gestión moderna de las entidades del Estado en Colombia, a partir de 1991, un componente muy valioso es el Control Interno, que es una herramienta que se ha desarrollado mediante los artículos 109 y 269 de la CPN. Su implementación se hace a través de la Ley 87 de 1993. En el desarrollo de esta ley, el sistema de control interno se centra en la gestión de lo público, mediante el autocontrol, que es un elemento importante para facilitar la gestión de las entidades públicas. Este sistema no cuenta con una herramienta técnica que permita verificar su desarrollo de una forma objetiva, ya que el evaluador final, que son aquellas personas que reciben los 
bienes o servicios elaborados por las entidades del Estado, no dispone de una herramienta que le permita plantear, en una forma técnica, objetiva y precisa, sus conceptos en relación con la gestión de las entidades del Estado.

\section{El Estado}

El Estado, como cualquier otra organización, dispone de recursos limitados; es decir, de su correcta planeación, dirección, ejecución y control, depende el éxito social que contribuya al bienestar de las comunidades residentes en el territorio. Como organización, el Estado está integrado por una sociedad que decide sobre la forma como organiza las relaciones para su convivencia dentro de dicho territorio. Según Ignacio Molina (1998), "el Estado es un concepto central de la Ciencia Política que designa la forma de organización jurídico-política con antonomasia. Surge en paralelo a la idea de soberanía y, etimológicamente, supone la plasmación estática de ésta" (p. 18). Esto equivale a decir que la soberanía es lo que va a facultar al pueblo para definir su tipo de organización y manejo de los recursos.

Un aspecto a tener en cuenta en el desarrollo de los estados modernos es el concepto de la Nueva Geografía Económica (NGE), cuya"idea central [...] es que el crecimiento regional obedece a una lógica de causación circular, en la que los encadenamientos hacia atrás y hacia delante de las empresas conducen a una aglomeración de actividades que se auto-refuerzan progresivamente" (Moncayo, 2003, p. 38).

Teniendo en cuenta este concepto, para que el país pueda participar activamente en el desarrollo de todo el movimiento global es necesario que la economía colombiana cuente con unas organizaciones públicas eficientes y dinámicas, capaces de dar una respuesta oportuna a la nueva realidad global, que faciliten el libre comercio y desarrollo de la empresa nacional.

Debido a la escasez de recursos, las empresas tratan de aprovechar al máximo los recursos con que cuentan. Es por ello que utilizan herramientas como la planeación estratégica, el balance Scorecard, la planeación por objetivos, entre 
otras, para que los gerentes cuenten con una ayuda técnica que soporte la toma de decisiones. La utilidad de estas herramientas depende, en muchas ocasiones, de la calidad de la información disponible.

Con base en lo anterior, se infiere que en la planeación que hagan los gerentes de las empresas de derecho privado, se deben tener en cuenta las relaciones con las entidades públicas. De acuerdo con los niveles de modernización tecnológica y operativa, estas entidades pueden soportar adecuadamente a las empresas privadas en la celeridad de los trámites que sean requeridos para el logro de sus objetivos.

Una de las complejidades que tienen las entidades del Estado es la apreciación generalizada de que sus procesos de control y evaluación, en muchas ocasiones, no corresponden con la realidad institucional, o con las pretensiones del orden institucional vigente. Esto ocasiona que los resultados de la gestión sean desdibujados ante la opinión pública, porque no se cuenta con un modelo que facilite el reconocimiento de la gestión del control que corresponda con las reales necesidades de la sociedad, y que solo se observe la gestión desde la óptica de los indicadores de gestión de los niveles de corrupción, y no de sus avances de acuerdo con la articulación de los planes de desarrollo propuestos, con la gestión de las organizaciones y la satisfacción de las necesidades de la sociedad.

\section{Modernización del Estado}

Teniendo como base la globalización y la interdependencia de los países, recientemente se han registrado aportes significativos para la modernización del Estado colombiano, lo cual ha tenido un impacto relevante en los roles y funciones que realiza. Lo anterior permite concebir al Estado actual como una organización con dinámicas transformadoras propias, como se ha evidenciado en el Estado colombiano durante las últimas décadas. 
A partir de 1991, el Estado cambia su rol, pasa de un Estado benefactor a un Estado garante, lo que conlleva la redefinición de su estructura administrativa, en la cual se identifican innovaciones en aspectos como intervención y gestión, lo que es conocido como "nueva gestión pública" (NGP). La nueva gestión pública se evidencia, a partir de 1980, como un nuevo modelo de administración pública con transformaciones que tienen repercusión en los gobiernos locales y sus relaciones con el entorno global. En este modelo, los Estados nacionales han perdido centralidad y el gobierno local se ve fortalecido, a través de un fenómeno denominado"nuevo localismo" (Brugué y Gomá, 1998).

En el ámbito latinoamericano, Colombia es uno de los países que ha experimentado transformaciones a partir de 1991, buscando mejorar la eficiencia de la gestión del Estado. En ese sentido se han implementado instrumentos de gestión como los siguientes:

- La participación ciudadana, el Art. 103 de la Constitución Política (C.P.)

- La descentralización administrativa, Art.1 de la C.P.

- Las reformas políticas de los partidos, Art. 107 de la C.P.

- El concepto de la función administrativa, Art. 209 de la C.P.

- La adopción del control interno para las entidades públicas, Art. 269 de C.P.

La incorporación de los anteriores aspectos en la Constitución Política de Colombia evidencia el desarrollo de la NGP para facilitar la gestión de las entidades. Esto además ha hecho que el Estado se modernice y armonice sus instituciones con base en las nuevas necesidades globales, que requieren de Estados que sean altamente eficientes, que faciliten los procesos comerciales al interior $y$ al exterior de los mismos. 


\section{Características de la Nueva Gestión Pública - NGP}

La NGP es un modelo que plantea que las entidades del Estado deben adoptar una serie de herramientas, procesos, valores y principios que han sido desarrollados con éxito en las empresas del sector privado. Lo anterior, con el fin de lograr que las empresas del sector público sean eficientes y que correspondan a las necesidades del sector productivo.

Los países que a partir de 1970 lideraron el proceso para mejorar la eficiencia de las empresas del Estado son Gran Bretaña, Australia, Nueva Zelanda y Estados Unidos. La NGP plantea el cambio en las estructuras y los procesos del sector público para que funcionen eficientemente, de manera similar como lo hacen las empresas privadas, en donde el fin es la satisfacción de las necesidades de los clientes.

La NGP procura el desarrollo de un Estado competitivo, tanto en lo local como en lo global, que realice las funciones básicas con una estructura pequeña que sea ágil, flexible y descentralizado. La optimización de estructuras, procesos y procedimientos de las organizaciones públicas busca aumentar la productividad de los servidores públicos, quienes deben cambiar el enfoque de las relaciones con el ciudadano y que de acuerdo con los postulados de la NGP deben redefinir el concepto de ciudadanos por el concepto de clientes.

A partir de trabajos como el de Osborne y Gaebler (1992), Estados Unidos divulgó la idea de la "reinvención del gobierno," bajo el concepto que un gobierno puede funcionar con tanta eficiencia y productividad como una empresa privada. Para lograr lo anterior es necesario ajustar su estructura para que esté sustentada en los resultados dentro del marco legal vigente.

En América Latina, en los años 80, países como Brasil, México, Chiley Argentina, han realizado intentos para implementar la NGP. En Colombia, a partir de la reforma constitucional de 1991, se inicia un proceso profundo de reestructuración de su administración pública, el cual cumple algunos de los lineamientos 
NGP. En esta reforma se establece un capítulo sobre la administración pública y otro sobre la función pública.

Es importante resaltar que el ajuste a la estructura de la administración pública colombiana se realizó mediante la expedición y promulgación de normas y decretos, basados en la Constitución de 1991, por ejemplo: la Ley 489 de 1998, que ordena la supresión y simplificación de trámites en la administración pública; la Ley 790 de 2002, que establece la fusión de entidades, organismos nacionales y ministerios; y la Ley 962 de 2005, que atiende la racionalización de trámites y procedimientos administrativos, con miras a facilitar la relación entre la ciudadanía y la administración.

La Constitución de 1991, la Ley 27 de1992 y la Ley 443 de 1998, tienen estrecha relación a la hora de asegurar que el Estado disponga de una mano de obra calificada para prestar el servicio público. Estas normas son relativas a la carrera administrativa, y en ellas se evidencian algunos de los principios de la NGP como son enganche, promoción y estabilidad, según el desempeño, los méritos, la medición y la premiación del funcionamiento individual y organizacional. Estos aspectos contribuyen a que las entidades del Estado puedan contar con personal altamente competitivo, acorde con las necesidades del sector privado.

De otra parte, la Ley 872 de 2003 crea el Sistema de Gestión de la Calidad de las Entidades del Estado, con el fin de mejorar y evaluar el desempeño institucional, con particular atención en la prestación de los servicios. Así mismo, mediante el decreto 1145 de 2004 se crea el Sistema General de Información Administrativa del Sector Público (SUIP), con el objetivo de realizar el seguimiento y la evaluación de la gestión pública en las entidades del Estado.

Finalmente, se identifica la presencia de la NGP en Colombia, con la incorporación de tecnologías de información para modernizar la administración del gobierno en línea o electrónico en las entidades de la rama ejecutiva nacional, lo que permite establecer que el estado está trabajando en mejorar la competitividad de sus entidades para que apoyen adecuadamente los procesos productivos de la economía. 


\section{El control}

Uno de los aspectos más significativos para el correcto funcionamiento de las organizaciones contemporáneas es el control, en ese sentido Ishikawa (1985, p. 71) comenta:

El Dr. Taylor solía describir el control con las palabras "planear, hacer, ver". ¿Qué significa "ver"? Para los alumnos de secundaria japoneses. Significa simplemente mirar algo. Esto no transmite el significado que Taylor le quiso dar. Por tanto, nosotros preferimos decir "planear, hacer, verificar, actuar". Esto es lo que llamamos Círculo de Control, y tenemos que hacerlo mover en la dirección correcta. Me ha parecido aconsejable redefinir este círculo dividiéndolo en seis categorías que ha demostrado su eficacia. Los seis pasos son los siguientes:

1. Determinar metas y objetivos.

2. Determinar métodos para alcanzar las metas.

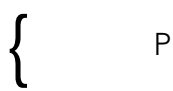

3. Dar educación y capacitación

4. Realizar el trabajo.

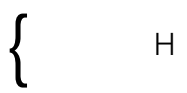

5. Verificar los efectos de la realización

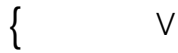

6. Emprender la acción apropiada

$\{\quad A(p .71)$

El concepto descrito por el Dr. Ishikawa constituye una propuesta de control muy completa, que en la actualidad tiene una constante aplicación, por ejemplo en las entidades del Estado, cuyo concepto se aplica en la NTGP-100-2009. El análisis inicial entregó como resultado una herramienta que, con su enfoque más amplio, es aplicable a todo tipo de organización, industria o proceso. La adecuada estructuración y puesta en marcha de este círculo de control debería propender por una cultura en todas las áreas y personas de una organización dirigida hacia su efectiva interacción y la generación de juicios críticos autónomos, sin que se deje de lado la apropiada supervisión.

Hoy en día, el control no se limita únicamente al ver la realización de una actividad o tarea asignada dentro de una jornada laboral o el desarrollo de una 
actividad en un cargo. El control se ha convertido en un estudio minucioso para identificar a dónde quiere llegar la organización, y, de acuerdo con los resultados alcanzados, realizar los análisis pertinentes para apoyar la toma de decisiones que se deben encaminar a la prevención de los riesgos para evitar el error.

El control es una de las principales actividades administrativas dentro de las organizaciones, de acuerdo con Koontz (1998), "es el proceso de verificar el desempeño de distintas áreas o funciones de una organización, usualmente implica una comparación entre un rendimiento esperado y uno observado, para verificar si se están cumpliendo los objetivos" (p. 109). El control es un componente esencial en el proceso administrativo de cualquier organización, dado que permite detectar lo que se está haciendo para cumplir los objetivos. Es una herramienta eficaz que permite mostrar resultados e incurrir en acciones de mejora en un proceso, cuando se detectan falencias. El control es importante hasta para la vida de las personas, porque permite medir los recursos y las acciones para la toma de decisiones asertivas que beneficien a la organización. El control permite visualizar más allá de las expectativas que se tengan para el cumplimiento de las metas. En una escala jerárquica esta herramienta permite estudiar los comportamientos de los subordinados y de la alta gerencia para estar a la vanguardia de los procesos y procedimientos en las organizaciones.

En las entidades del Estado, el personal encargado de dirigir las funciones y actividades de un proceso, debe tener claro que el control es una de las prioridades para evidenciar si realmente se está llevando a cabo lo planteado en las políticas y objetivos. Fred R. (2008) afirma que "el control comprende aquellas actividades emprendidas para asegurar que las operaciones reales se ajusten a las planeadas" (p. 135). Cuando las empresas del estado realizan un adecuado proceso de planeación, el control se puede realizar cotejando los resultados obtenidos con lo planeado, lo que facilita procesos de análisis y evaluación a profundidad. De esta forma los resultados obtenidos permiten enfocarse en puntos críticos y realizar procedimientos de retroalimentación adecuados y oportunos. 
La función del control brinda la ocasión perfecta para analizar a profundidad lo que se ha establecido en la planeación . En estos casos, Fred R. (2008) comenta que "el control consta de pasos fundamentales: 1. Establecer normas de desempeño. 2. Medir el desempeño individual y de la organización. 3. Comparar el desempeño real con las normas del desempeño establecido. 4. Emprender acciones correctivas" (p. 156). Así mismo, se deben realizar mediciones de información, examinando situaciones en las que los resultados pueden llegar a ser positivos o negativos; este análisis permite tomar decisiones que conlleven situaciones inesperadas o reacciones por parte del personal subordinado. Para tal caso, la entidad debe ofrecer, con anterioridad, capacitación al personal y orientación para saber afrontar las observaciones o cambios necesarios para mejorar los procedimientos y los resultados.

\section{El control interno}

A partir de la Constitución Política de 1991, el control interno ha tenido un desarrollo normativo que ha permitido su implementación y desarrollo. Así, por ejemplo, los artículos 209 y 269 de la Constitución dan origen al control interno en el Estado colombiano, en estos se establecen los principios que rigen la función administrativa y el control interno. De esta forma, el control interno constituye un avance importante que apoya y facilita la gestión de las entidades del Estado, partiendo del autocontrol, con el fin de lograr el uso adecuado de los recursos del erario.

La Ley 87 de 1993 reglamenta el Sistema de Control Interno (SCI), mediante la directiva presidencial oo2 de 1994, que establece los lineamientos del (SCI), generando un control que agiliza la gestión de las entidades públicas para que puedan soportar, de forma adecuada, la gestión del sector empresarial.

Una de las necesidades de los Estados modernos es contar con aparatos administrativos que respondan a las nuevas necesidades de los mercados globales. Por esta razón, "la necesidad de volver eficientes y eficaces a las instituciones del Estado [colombiano] hizo que la administración pública en general volviera 
su mirada hacia las teorías administrativas modernas... entre ellas los controles que deben tener en toda la organización" (Charry, 1996, p. 17). Lo anterior, con el fin de contar con instituciones que den pronta respuesta a las necesidades de la sociedad y de las empresas.

La Constitución Política, en los artículos 209 y 269, establece que la administración pública deberá desarrollarse bajo los principios de igualdad, moralidad, eficacia, economía, celeridad, imparcialidad y publicidad. En consecuencia, las entidades públicas deben diseñar y aplicar, según la naturaleza de sus funciones, los métodos y procedimientos de control interno y a su vez diseñar e implementar el sistema de evaluación de gestión y resultados.

La constitución, en su artículo 267, eliminó el ejercicio del control previo que realizaban las contralorías, al considerarse que estos hacían lento los procesos en las entidades públicas, reemplazándolo por un control posterior y selectivo, con el fin de vigilar y evaluar la gestión fiscal del Estado y de los particulares o entidades que manejen fondos o bienes de la nación. En 1993, a través de las leyes 87 y 42, se produce el desarrollo normativo general de las disposiciones constitucionales. La Ley 42 de 1993 da a las contralorías la función de vigilar al Estado a través de la implementación de los controles financieros, de legalidad de gestión, de resultados y la evaluación de los sistemas de control interno de cada entidad.

\section{La Ley 87 de 1993}

La Ley 87 de 1993 estructura la concepción de control, la cual se aclara a través de la directiva presidencial 002 de 1994, generando un control que agilice la gestión de las entidades públicas, que pueda soportar en forma adecuada la gestión del sector empresarial en su relación con el sector externo. Además, establece las normas para el ejercicio de control interno en las entidades y organismos del Estado. Así mismo, con el decreto 1826 y la directiva presidencial O2 de 1994, se concretan acciones por el presidente de la República, para posibilitar la implementación del Sistema de Control Interno, mediante la creación 
de las Oficinas de Control Interno como instancia de retroalimentación al interior de cada entidad del Estado.

La Ley 489 de 1998, Estatuto básico de la administración pública, crea el sistema Nacional de Control Interno como una instancia de articulación del sistema en todo el Estado, bajo la coordinación del presidente de la república, como máxima autoridad administrativa.

Mediante el decreto reglamentario 2145 de 1999 se consolida el papel de las diferentes instancias en materia de control interno dentro del sistema nacional. Se establece la obligatoriedad de presentar informes al consejo asesor, a través de un informe anual sobre el avance del sistema de control interno institucional. Con la directiva o4 de octubre de 2007 se adopta una política presidencial para garantizar la independencia y la objetividad de los jefes de oficina de control interno o quien haga sus veces.

La Ley 734 de 2002, por la cual se expide el nuevo código disciplinario único, contempla como deber de todos los servidores públicos adoptar el sistema de control interno y la función, independiente de auditoría interna de que trata la ley 87 de 1993 y demás normas que modifique o complementen.

La Ley 872 de 2003, por medio de la cual se crea el sistema de gestión de calidad en la rama ejecutiva del poder público, como herramienta de gestión sistemática y transparente que permita dirigir y evaluar el desempeño institucional, en términos de calidad y satisfacción social en la prestación de servicios a cargo de las entidades. De acuerdo con lo planteado por Charry (1996), se puede concluir que "el control de gestión es un instrumento que mide la eficiencia con la que se han adelantado las metas, planes y objetivo de una institución, permite deducir que no existen desviaciones entre lo planeado y lo ejecutado" (p. 27). Esto convierte al gerente público en un actor principal en el proceso administrativo para controlar los procesos y el cumplimiento de las metas en la organización. 
A lo anterior se suman: el decreto 4110 de 2004, que reglamenta la Ley 872 y se adopta a la Norma Técnica de Calidad en la Gestión Pública; el decreto 1599 de 2005, por medio del cual se adopta el Modelo Estándar de Control Interno para el Estado Colombiano, conocido como MECl; junto con el decreto 943 de 2014, por el cual se actualiza el modelo estándar de control interno.

Igualmente, el sistema de control interno fue modernizado mediante la Ley 1499 de 2017, en donde se articulan los sistemas de control de gestión con el sistema de control interno, lo que ha permitido una actualización del Modelo Estándar de Control Interno (MECl) y el Sistema de Control Interno. Este instrumento apunta a mejorar la competitividad de las entidades del Estado, acorde con las crecientes necesidades de la competitividad global y local, que requieren de entidades públicas que soporten de manera adecuada los procesos en los que interactúan con las empresas de derecho privado.

\section{Propuestas y recomendaciones}

Para modernizar las herramientas de gestión del Estado, acorde con la globalidad, es necesario implementar instrumentos de gestión que se han venido desarrollando en las empresas del sector privado.

En procura de consolidar empresas públicas altamente eficientes que desarroIlen esfuerzos para mejorar su competitividad, como soporte para el desarrollo de las empresas del sector privado, se deben implementar herramientas como:

- El enfoque sistémico.

- La vigilancia tecnológica.

- La inteligencia competitiva.

- Los factores críticos de vigilancia. 


\section{Enfoque sistémico}

En el documento de las organizaciones y los métodos de su entendimiento, elaborado por Alexandra Montoya Restrepo e Iván Alonso Montoya Restrepo, se hace una comparación de la administración con otras ciencias. Allí se plantea que, a través de la similitud de las organizaciones con los organismos vivos, que estudia la biótica, se pueden desarrollar procesos de enseñanza y aprendizaje sobre los procesos que se realizan en la evolución de las organizaciones.

Uno de los principales temas planteados es que existen diversas formas para realizar comparaciones; según Stafford Beer, "existen varios niveles de comparación en los que el científico podría tratar de trabajar" (citado por Montoya, 2003, p. 2). De acuerdo con lo anterior se establece que existen múltiples niveles de comparación, en los que cualquier científico puede trabajar para desarrollar sus procesos de análisis de los diferentes objetos que desea analizar, entre ellos está la evolución y la gestión de las organizaciones.

Para realizar la observación los científicos pueden utilizar la metáfora. De acuerdo con el planteamiento de Montoya, la "metáfora es un recurso poético y su fuerza es estética; no puede ofrecer a la ciencia más ayuda que su facilidad verbal" (2003, p. 2), aspecto que ayuda al científico a plasmar en una forma concreta y organizada los procesos que ha podido observar a lo largo de su proceso de estudio sobre el objeto observado.

Otro mecanismo de comparación es la analogía, que permite observar, dentro de un concepto de biótica, el comportamiento de las organizaciones comparándolas con el comportamiento de los seres vivos y de esta forma entender su evolución y desarrollo. Para Beer"si dos cosas son literalmente idénticas entre sí, las conclusiones que son válidas para una de ellas, seguramente lo son para la otra, en condiciones similares" (citado por Montoya, 2003, p. 120); de lo cual se deduce que la analogía es una herramienta útil para la comparación, pero sus resultados no son absolutos, ya que estos dependen de las condiciones en donde se desempeñan los organismos vivos y las organizaciones. 
Otro de los instrumentos que pueden ser utilizados por los investigadores es la metáfora. Morgan la define como "un modo de pensar y un modo de ver que traspasa el cómo se comprende el mundo real" (2003, p.3). Lo que permite que el investigador pueda exponer en la metáfora sus diferentes planteamientos sobre las observaciones realizadas.

Uno de los aspectos significativos que plantea Stanfor Beer sobre el control es "que el sistema de control de la empresa era como el sistema nervioso central del cuerpo humano" (2003, p. 8), lo que permite inferir que esta analogía es una reflexión sobre la importancia que tiene para el cuerpo humano su sistema nervioso. La misma importancia deben tener para las organizaciones los sistemas de control, que contribuyen a su buen funcionamiento.

Mediante el uso de la analogía se puede observar la organización como un organismo, ya que las organizaciones están conformadas por partes y elementos, lo que permite no solo observar y analizar su funcionamiento, sino hacer un símil con el objeto de estudio de la biótica que, mediante la biología, estudia las células, los órganos, y los organismos como elementos articulados en un sistema que garantiza la pervivencia de los seres vivos, aspecto muy parecido a las organizaciones artificiales o empresas.

La investigación, titulada El poder de conocimiento tácito: por encima del aprendizaje Organizacional, parte de una comprensión del comportamiento de un vendedor de una empresa, con el fin de establecer el arquetipo del vendedor en cada una de sus etapas de evolución en una organización. Comienza con una metáfora comparativa entre el procedimiento y la espontaneidad, con el fin de dilucidar la dicotomía que puede existir entre estos términos, para lo cual inicia con el planteamiento de la pertinencia sobre el conocimiento tácito y explícito sobre los procesos de venta.

Con respecto al análisis del procedimiento, Arbonies (2004) señala que"el conocimiento tácito es aquel que aun poseyéndolo difícilmente podemos explicitar. Es subjetivo y está basado en la experiencia personal y fuertemente ligado a la acción de los individuos en un contexto determinado " (p. 2). Lo que permite 
inferir que, aunque los procedimientos son importantes en la gestión de un proceso, no necesariamente puede ser la solución en determinados momentos en los se requiere la experiencia. En casos determinados, se puede plantear que un vendedor junior que se apegue al cumplimiento de los procedimientos, difícilmente puede superar la experiencia en las ventas de un vendedor sénior. De ahí que se debe reflexionar sobre la importancia del conocimiento tácito que se ha desarrollado a través del paso del tiempo, que le da competencias adicionales a la que se da con el conocimiento explícito en los manuales de funciones de las empresas. En otras palabras, el conocimiento tácito es aquel que tienen las personas, en la mayoría de las cosas, sin saber que lo tienen; este tipo de conocimiento les permite tener competencias más desarrolladas en un tema específico que otras personas.

Muchos funcionarios públicos pueden tener un conocimiento tácito en el control que ejercen sobre las funciones que realizan, pero muchas veces no son conscientes de tener este conocimiento; lo que hace que tengan que recurrir a nuevos procesos de control interno o a controles externos, generando demoras innecesarias en la atención de los requerimientos del cliente externo. Este aspecto afecta la gestión de los servidores públicos y genera una distorsión de la calidad de los servicios, por parte de los ciudadanos que usan los mismos.

De acuerdo con lo observado, el investigador que escoja como objeto de observación la empresa se puede apoyar en la metáfora, la analogía y el símil como herramientas que le permitan comparar la organización empresarial con los seres vivos. Las conclusiones que son válidas en los organismos vivos pueden ser válidas para las empresas. Por esta razón se hará un análisis en el planteamiento del modelo, que corresponde a elementos bióticos que permiten corroborar la necesidad de plantear un nuevo elemento que complemente al actual Sistema de Control Interno implementado en el Estado. 


\section{Vigilancia tecnológica}

Una de las características de la producción de bienes y servicios de manera global, ya sea por empresas particulares, públicas o mixtas, es que esta se enmarca dentro de las condiciones naturales propias de cada país. Esto ocasiona que determinadas economías, aprovechando sus ventajas comparativas, se dediquen a la producción de determinados bienes o servicios, con el fin de aprovechar al máximo las ventajas de que disponen y así obtener una mayor productividad. Mankiw plantea que la"productividad es la cantidad de bienes y servicios producidos en cada hora de trabajo realizada" (1998, p. 51), lo que lleva a los diferentes actores económicos a tratar de ser productivos aprovechando al máximo las condiciones naturales disponibles. En ese sentido, las empresas (públicas y privadas) hoy día deben utilizar la inteligencia competitiva para poder ganar espacios en los mercados y satisfacer las necesidades de la sociedad.

Las organizaciones en Colombia, tanto públicas como privadas, están obligadas a emprender el camino hacia la competitividad para que puedan crecer en el contexto local e internacional, en nuevos mercados en el marco de los convenios comerciales unilaterales y multilaterales suscritos entre el país y otros industrializados o de economías emergentes.

La gestión tecnológica se define como la estrategia de la empresa que se encarga de identificar, analizar, planear e implantar desarrollos y aplicaciones tecnológicas, tendientes a mejorar sustancialmente el rendimiento de los procesos del negocio, generando diferenciación a partir del valor agregado competitivo (Sánchez, 2002). Lo que permite inferir que para el desarrollo de organizaciones competitivas es necesario implementar un buen proceso de gestión tecnológica.

Actualmente las organizaciones empresariales deben invertir en el desarrollo científico, o por lo menos tecnológico, que demanda el contexto de la empresa y los servicios que ofrece, siendo este un baluarte comparativo y competitivo en el sector económico en el que hace presencia. Solo de esta forma podrá tener un mayor conocimiento del contexto en donde participa. 
Vale la pena recordar que la inteligencia tecnológica ha tenido una evolución, representada en aportes teóricos de países como Estados Unidos (Michael Porter, 1986; Robert Gates, ex director de la CIA, 1992; Bill Clinton, 1996 y Advocacy Center); Japón (Miti y Jetro, 1950); Italia (Edouard Balladur, Bernard Carayon, Alain Juillet, 1995), entre otros. La Unión Europea fue la primera comunidad de Estados que adoptó la inteligencia económica como uno de los vectores importantes de política de competitividad industrial (Philippe Clero, Universidad de París II, Inteligencia económica: retos actuales y perspectivas, 1997).

Las orientaciones tácitas en el mundo empresarial obligan a hacer un análisis reflexivo por parte de los dirigentes y empresarios en el país. Si la Unión Europea le apuesta a la inteligencia tecnológica, permitiendo que sus empresas se especialicen y sean mucho más efectivas en sus procesos, obtendrá un nivel de posicionamiento estratégico y competitivo, obligándolas a crecer hasta el punto de hacerlas multinacionales o transnacionales. ¿Por qué no hacer lo mismo en Colombia?

En Colombia se deben estimular los procesos de investigación en adelantos científicos y tecnológicos con el fin de incursionar en la cultura de la economía competitiva basada en el conocimiento, desde la óptica de Estado nación, fortaleciendo el entorno legal y las entidades públicas y privadas para fomentar la colaboración y el desarrollo desde sus sectores. Los empresarios en colombianos deben orientar sus objetivos a:

- Promover la capacidad innovadora de las empresas

- Sensibilizar a los socios o accionistas en la importancia de la protección de su patrimonio

- Hacer esfuerzos en procesos de investigación disciplinar de las empresas

- Actualizar su información veraz y oportunamente

- Desarrollar programas de capacitación y formación continua de punta y con prospectiva del negocio. 
Existen grandes barreras en el proceso de consecución de información para lograr identificar la realidad de los avances tecnológicos. Y esta vigilancia no es tarea fácil, puesto que gran parte de la misma no está documentada ni validada, es de carácter subjetivo, o incluso en algunos casos se debe generar. Uno de los grandes problemas no es la consecución de la información sino el tratamiento de la misma.

En algunos momentos daría la impresión de construir un gran rompecabezas, ya que en este proceso se incluye la necesidad de identificar, recolectar, procesar y analizar información que permita obtener conclusiones de base para desarrollar estrategias e inteligencia tecnológica en la proyección prospectiva de las organizaciones.

Los procesos de inteligencia comprenden básicamente las siguientes etapas:

1). Identificación de las necesidades del cliente.

2). Planificación y dirección.

3). Recolección de la información.

4). Análisis de la información.

5). Transmisión del conocimiento generado.

Una de las grandes dificultades es contar con las personas adecuadas para desarrollar estas investigaciones. Sujetos que tengan la habilidad de consultar fuentes primarias y secundarias, que puedan producir inteligencia analítica para las personas encargadas de la toma de decisión y directivos u operativos, promoviendo elementos para comprender las ambigüedades y la deficiencia de información en los desarrollos externos, que son base de la competitividad de las empresas y el éxito de los negocios.

El camino por recorrer en torno a la sensibilización de los dirigentes y empresarios colombianos es largo. En el contexto actual de convenios comerciales, en lo que tiene que ver con tratados de libre comercio con países industrializados o emergentes, Colombia evidencia que las empresas que no están dispuestas a invertir e implementar estrategias de inteligencia no van a perdurar en el 
mercado y su futuro será incierto; además, si las organizaciones del Estado no están debidamente preparadas serán un lastre que genere lentitud en los procesos de las empresas globales.

\section{La inteligencia competitiva}

La administración de las organizaciones requiere de niveles de profesionalización elevados. De esta forma, el profesional que realice la actividad debe hacer un uso adecuado de los recursos de la empresa, propendiendo por su optimización en el logro del objetivo empresarial.

Uno de los recursos importantes en las organizaciones públicas o privadas es el manejo adecuado y oportuno de la información disponible. En ese sentido, es importante analizar cómo se puede recolectar la información y cuál será el mecanismo más adecuado para el almacenamiento y procesamiento de la misma, con el único fin de desarrollar en las organizaciones la Inteligencia Competitiva (IC). No basta solo con tener equipos de cómputo para el procesamiento de la información, pues también se hace necesario escoger los equipos adecuados que correspondan a las necesidades de la organización.

De acuerdo con lo esbozado, la administración eficiente de las organizaciones debe desarrollar unas competencias específicas, como la de identificar el hardware y software necesarios para la operación, así como la información, interna y externa, que le sirva para ser más productiva y desarrollar sus procesos con eficiencia, en cumplimiento de la misión institucional confiada por el Estado en desarrollo de la función administrativa.

Para desarrollar las competencias reseñadas, las empresas del Estado deben generar análisis reflexivos, críticos y comparativos que les permitan estar en capacidad de seleccionar los principales recursos tecnológicos y bases de datos que permitan el fortalecimiento de las organizaciones. De esta forma se constituirán como empresas públicas con elevados niveles de modernización, que les permitan una participación exitosa en el mercado. 
Uno de los objetivos de las organizaciones modernas, entre ellas las entidades del Estado, es el aprovechamiento de las relaciones entre la gestión tecnológica y la gestión organizacional, aspecto trascendental para la toma adecuada de decisiones como un ejercicio natural de la Inteligencia Competitiva (IC) en la organización.

Otro de los objetivos específicos es cómo la organización puede desarrollar el conocimiento entorno a la IC y la gestión tecnológica, aspectos que ayudarán a que la empresa alcance elevados niveles de competitividad organizacional. Otro de los elementos relevantes es la constante actualización del conocimiento sobre los cambios tecnológicos y su aplicación en la empresa moderna, lo que hace que el Estado moderno tenga unas empresas públicas que deben contar con los desarrollos tecnológicos necesarios para enfrentar las nuevas condiciones sociales y del mercado, con el fin de prestar servicios a la sociedad, ya sea como ciudadanos o mediante empresas, con los más altos niveles de eficiencia, eficacia y economía, que garanticen la evolución de la economía como un referente del crecimiento económico.

\section{Los Factores Críticos de Vigilancia (FCV)}

Las empresas que desarrollan modelos de gestión, centrados en la inteligencia competitiva, los implementan con el fin de contar con una herramienta que les permita tomar decisiones soportadas en la mayor información posible. Por eso desean conocer cómo se comporta el entorno observando la evolución de los productos, la competencia, los clientes, los procesos de mercadeo, entre muchas otras variables que son de interés de la organización y que en un momento determinado pueden afectar a la empresa.

El desarrollo de los FCV, dentro de un sistema de vigilancia tecnológica, debe corresponder a las necesidades de información. Por este motivo, la empresa debe reflexionar sobre qué debe monitorear, con qué periodicidad, qué tipo de informe debe obtener y qué clientes internos son los usuarios de esta in- 
formación. De acuerdo con las necesidades empresariales se han desarrollado diversos modelos para organizar los FCV como los siguientes:

- Carácter temporal: este modelo analiza el comportamiento de los aspectos a monitorear en la vigilancia tecnológica.

- Carácter arborescente: consiste en que los FCV se clasifican de acuerdo con la importancia y la dependencia que el usuario dé a la información recolectada.

- Vigilancia en función del grado de relevancia en la organización, pública o privada: consiste en identificar qué tipo de tecnología afecta a la organización, qué nivel de dependencia se tiene, qué indicadores sirven para identificar y monitorear los FCV y a partir de esta información adecuar la vigilancia tecnológica, de acuerdo con los intereses de la organización.

- Enfoque colectivo de vigilancia: es cuando se opta por mantener una vigilancia sobre un grupo de empresas de una región o un país, generalmente se centran en desarrollar los determinantes definidos como los recursos, el mercado, los sectores relacionados y las estructuras de las empresas con las cuales se compite.

Las empresas del Estado deben desarrollar un modelo de Gestión Estratégica Tecnológica - GET eficiente, que les permita anticiparse a las oportunidades y amenazas presentes en el entorno, con base en la organización adecuada de un proceso de vigilancia tecnológica e inteligencia competitiva. Por esta razón, se hará una reflexión del modelo de Gestión Estratégica Tecnológica GET, propuesto por Álvaro Pedroza (2001), el cual está enfocado en el desarrollo de un modelo de cinco etapas, en las cuales se pretende ligar la estrategia tecnológica de las empresas con la estrategia corporativa en sí.

El modelo propuesto por Pedroza (2001) inicia con la determinación de los pares mercado/producto de interés, en la cual se pueden utilizar herramientas matriciales tales como la matriz de información de las líneas del producto, en la que se detallan aspectos tales como: categorías de productos, categorías de mercados, año de introducción del producto, ventas promedio en los últi- 
mos tres años y porcentaje de las ventas totales de la compañía. Así mismo, se utilizan matrices de análisis mercado-producto, las cuales muestran los segmentos de mercado atendidos por los productos que ofrecen las empresas, en términos de ventas totales, tasa anual de crecimiento, porcentaje de contribución a las utilidades y porcentaje de participación en el segmento del mercado en particular, en cuyas celdas prioritarias se encuentran relacionados otros factores tales como el nivel de dificultad de ganarle a los competidores, crecimiento del mercado de sus clientes e intuición.

El empleo de herramientas de gestión tecnológica, tales como los árboles tecnológicos, es de gran utilidad en una segunda etapa, ya que permite evaluar el significado de cualquier movimiento o desarrollo tecnológico de los competidores. Para el caso de las necesidades de las empresas del Estado, es necesario concentrarse en los aspectos más relevantes para la definición de los FCV, los cuales pueden resumirse en el cuestionamiento y reflexión profunda de los siguientes ítems a monitorear para mejorar la gestión de las entidades.

En cuanto a la definición de las tecnologías necesarias, es importante evaluar el tipo de tecnología - equipo, producto, proceso, operación, gestión administrativa, comportamiento humano - frente al desarrollo de la tecnología original, la disponibilidad de dicha tecnología, los mecanismos de transferencia y la adaptabilidad, como tal. La tercera etapa del modelo propuesto consiste en la evaluación de las necesidades de desarrollo de competencias tecnológicas, en la cual se analizan las siguientes dimensiones presentes en la empresa, con respecto a las tecnologías con las que cuenta cada organización: presupuesto de investigación y desarrollo, gastos en otras actividades técnicas, recursos humanos, equipamiento y laboratorios, patentes, distribución del presupuesto para mejoramiento de producto existente, desarrollo de nuevos productos para la empresa, desarrollo de nuevos productos para la industria (Pedroza, 2001).

Después de la evaluación dada en la tercera etapa, se procede a elegir los proyectos tecnológicos, acordes con las necesidades de la empresa, de los cuales se pueden distinguir dos tipologías: proyectos de investigación, desarrollo e 
ingeniería relacionados con productos o procesos, y proyectos de inversión de capital. En este aspecto es importante evaluar cada una de las necesidades tecnológicas previamente identificadas, con las tecnologías críticas o clave, el tipo de proyecto tecnológico, los costos y los beneficios; de igual forma, es necesario considerar los siguientes criterios para la evaluación de dichos proyectos, los cuales pueden ser: riesgo tecnológico, riesgo comercial, retorno, impacto competitivo, consistencia con la prioridad producto/sociedad, consistencia con la estrategia y las necesidades de la sociedad, consistencia con las tecnologías medulares, posibilidad de patentar, consistencia con las oportunidades y amenazas tecnológicas, duración, costo, tiempo e inversiones post-proyecto.

\section{Conclusiones}

Para el desarrollo de las herramientas de la NGP, en especial el autocontrol, con la implementación de la nueva tecnología administrativa, el Estado debe iniciar procesos que apunten a su desarrollo y a su vez sus procesos generen las condiciones idóneas para que las empresas del sector privado puedan ser más competitivas tanto en el contexto global como local.

El desarrollo del control interno, como herramienta de gestión, con una tecnología de punta, que utilice las más recientes técnicas apropiadas por el sector privado, facilitarían el proceso para que las empresas públicas se conviertan en un eslabón muy importante para el desarrollo de la competitividad del sector productivo de la economía colombiana.

Mediante la aplicación de políticas adecuadas, se pueden generar las condiciones propicias para el desarrollo de sus economías, siempre propendiendo por un bienestar de los ciudadanos, que le confían al estado la administración de los bienes públicos. Teniendo en cuenta las nuevas teorías de desarrollo endógeno, las cuales sustentan la idea de que los países o regiones encierran en su propia dinámica la clave para un crecimiento sostenido. 
Las empresas modernas tienen un gran contacto con el Estado, se observa la relación de pago de tributos como el impuesto a la renta, el impuesto de avisos, tableros y complementarios, cuando son importadores los diferentes impuestos para la nacionalización de mercancías, y cuando son exportadores deben tener presente los trámites y tributos para la exportación de bienes o servicios según sea las necesidades de los empresarios, es por este motivo que para que exista una relación armoniosa entre las empresas y el Estado, se deben desarrollar los procesos administrativos con la mayor eficiencia y evitar que los errores generen traumatismos en la prestación de los servicios, por ello es necesario que el Estado cuente con mecanismos de control que no generen traumatismos y que faciliten la gestión de la entidades que lo conforman.

En la sociedad contemporánea el empresario no puede olvidar la existencia del Estado en el ejercicio de su actividad diaria o en el diseño de su estrategia: las formas de actuación del Estado cambian con el tiempo, pero todo parece indicar que el Estado mismo no desaparece. Al contrario, en las últimas décadas, su peso no ha hecho más que crecer; y es lógico que se cuestione si ese crecimiento tiene límites, y, si los tiene, dónde están. Para ello hay que preguntarse cuál es la razón de ser de las diversas actividades del Estado. En la práctica, en las economías de mercado, se observa que las relaciones entre el sector público y el empresario privado abarcan una amplia gama de sentimientos que van de la cordialidad, e incluso la complicidad, al enfrentamiento, pasando por la indiferencia. Que esas relaciones sean de uno u otro carácter depende de muchos factores: de la actividad de que se trate (que puede merecer más o menos atención por parte de los poderes públicos) del partido que gobierne aunque, e incluso del carácter del empresario o del funcionario que le haya tocado en suerte. Se habla a menudo de la conveniencia de que Estado y empresa trabajen juntos, pero esto no siempre es posible. Esta situación se replica en la mayoría de los países en lo que concierne a la relación Estado-empresa, lo que hace que surja un tema crítico que tiene que ver con la fiscalización y el control de las entidades públicas.

La relación de la empresa con el Estado se hace más evidente cuando se observa la regulación que existe para la contratación y el pago de la mano de obra 
que participa en el proceso productico. Además de la regulación sobre patentes y su cumplimiento dentro del contexto nacional. Estos son unos cuantos ejemplos de la estrecha relación entre el Estado y las empresas privadas.

De acuerdo con lo planteado es muy importante que los empresarios, miembros integrantes de la sociedad, en el momento de la toma de decisiones tengan en cuenta los efectos que pueden tener estas en el actual orden constitucional, con el fin de que puedan aprovechar adecuadamente las ventajas que ha establecido el Estado colombiano para el desarrollo de la libre empresa y la protección a la propiedad privada, y evitarse que por desconocimiento de las relaciones con el Estado se tomen decisiones cuyos efectos sean negativos para la sociedad y por ende genere una sanción de parte del Estado.

Cuando los empresarios tienen pleno conocimiento de las actividades del Estado y de sus procesos, y las relaciones son claras, es posible que éstos puedan participar, con gran probabilidad de tener éxito, en los diferentes procesos licitatorios que adelanta el gobierno para la adquisición de bienes y servicios y de esta forma contribuir a que el Estado pueda cumplir con la función que ha sido establecida en la Constitución Política de Colombia. Es por este motivo que el Estado debe desarrollar unos procesos con eficiencia en claro interés de beneficiar el desempeño de las empresas privadas.

\section{Referencias}

Arbonies O. y Calzada, M. (2004). El poder del conocimiento tácito por encima del aprendizaje organizacional. Intangible Capital, 6.

Brugué, Q. y Ricard, G. (coords), (1998). Gobiernos locales y Políticas públicas. Barcelona: Ariel Ciencia Política.

Charry, R. (1996). Los indicadores de gestión y de resultados en las entidades del Estado. Medellín, Colombia: Asociación Colombiana de Administradores Públicos. 
Congreso de la República, Ley 443 de 1998.

Congreso de la República, Ley 962 de 2005.

Congreso de la República, Ley 27 de1992.

Congreso de la República, Ley 489 de 1998.

Congreso de la República, Ley 790 de 2002.

Congreso de la República, Ley 87 de 1993.

Congreso de la República, Ley 1499 del 2017.

Constitución Política de Colombia. 1991,

Directiva presidencial, 002 de 1994.

Escorsa. P. (2007). ¿Qué es la Inteligencia Competitiva? Conferencia internacional sobre inteligencia competitiva, Disponible en http://www.madrimasd.org/informacionidi/ agenda/inteligencia-competitiva/documentos/pere_escorsa.pdf

Fred, R. (2008). Conceptos de Administración Estratégica. Décimo primera edición. México: Pearson educación.

Ishikawa. K. (1995). La Esencia del Control de Calidad. Disponible en http://jrvargas.files. wordpress.com/2011/02/que_es_el_control_total_de_lacalidad_-_kauro_ishikawa.pdf.

Koontz, H. (1998). Administración una perspectiva global. México: McGraw-Hill..

Mankiw N. G., (1998). Principios de Macroeconomía. Mc Graw-Hill, Madrid.

Molina, I. (1998). Conceptos fundamentales de Ciencia Política. Alianza Editorial. Madrid. 
Moncayo, J, E. (2003). Nuevas Teorías y Enfoques Conceptuales Sobre el Desarrollo Regional: ¿Hacia un Nuevo Paradigma? Revista de Economía Institucional, Vol. 5, No. 8 Primer semestre 2013.

Montoya A. y Motoya I. (2003). Las organizaciones y los métodos de su entendimiento. Innovar. Univesidad Nacional.

Osborne, D. y Gaebler, T. (1992). La reinvención del gobierno. Buenos Aires: Paidós.

Pedroza, A. (2001). Modelo para la Gestión Estratégica de Tecnología (GET). Universidad Autónoma de México (UAM). 